\title{
Structural changes in a Schiff base molecular assembly initiated by scanning tunneling microscopy tip
}

\author{
A Tomak ${ }^{1}$, C Bacaksiz ${ }^{2}$, G Mendirek ${ }^{3}$, H Sahin ${ }^{4,5}$, D Hur ${ }^{6}$, K Görgün ${ }^{7}$, \\ R T Senger ${ }^{2}$, Ö Birer ${ }^{8}$, F M Peeters ${ }^{4}$ and H M Zareie ${ }^{1,9}$ \\ ${ }^{1}$ Department of Materials Science and Engineering, Izmir Institute of Technology, Izmir 35430, Turkey \\ ${ }^{2}$ Department of Physics, Izmir Institute of Technology, Izmir 35430, Turkey \\ ${ }^{3}$ Biotechnology and Bioengineering Graduate Programme, Izmir Institute of Technology, Izmir 35430, \\ Turkey \\ ${ }^{4}$ Department of Physics, University of Antwerp, Groenenborgerlaan 171, 2020 Antwerpen, Belgium \\ ${ }^{5}$ Department of Photonics, Izmir Institute of Technology, Izmir 35430, Turkey \\ ${ }^{6}$ Plant, Drug and Scientific Research Center (BIBAM), Anadolu University, Eskişehir 26470, Turkey \\ ${ }^{7}$ Department of Chemistry, Eskişehir Osmangazi University, Eskişehir 26480, Turkey \\ ${ }^{8}$ Chemistry Department, KUYTAM Surface Science and Technology Center, Koc University, Istanbul \\ 34450, Turkey \\ ${ }^{9}$ Microstructural Analysis Unit, School of Physics and Advanced Materials, University of Technology, \\ Sydney, Ultimo NSW 2007, Australia \\ E-mail: hadizareie@iyte.edu.tr
}

Received 8 April 2016, revised 17 May 2016

Accepted for publication 31 May 2016

Published 4 July 2016

\begin{abstract}
We report the controlled self-organization and switching of newly designed Schiff base (E)-4-((4(phenylethynyl) benzylidene) amino) benzenethiol (EPBB) molecules on a Au (111) surface at room temperature. Scanning tunneling microscopy and spectroscopy (STM/STS) were used to image and analyze the conformational changes of the EPBB molecules. The conformational change of the molecules was induced by using the STM tip while increasing the tunneling current. The switching of a domain or island of molecules was shown to be induced by the STM tip during scanning. Unambiguous fingerprints of the switching mechanism were observed via STM/STS measurements. Surface-enhanced Raman scattering was employed, to control and identify quantitatively the switching mechanism of molecules in a monolayer. Density functional theory calculations were also performed in order to understand the microscopic details of the switching mechanism. These calculations revealed that the molecular switching behavior stemmed from the strong interaction of the EPBB molecules with the STM tip. Our approach to controlling intermolecular mechanics provides a path towards the bottom-up assembly of more sophisticated molecular machines.
\end{abstract}

S Online supplementary data available from stacks.iop.org/NANO/27/335601/mmedia

Keywords: Schiff base molecule, self-assembled monolayers, scanning tunneling microscopy, switches, DFT, SERS

(Some figures may appear in colour only in the online journal)

\section{Introduction}

The accurate positioning and movement of components within the nanoscale range considerably enhance the capabilities of nanodevices in numerous applications and also in yet-to-be-discovered new applications [1]. There is considerable optimism that molecular switches will play a role in the design and operation of nanoscale electronic devices [2,3] 
and a plethora of molecular switches have been reported in the literature reflecting the technological importance of these devices. There are many molecular properties amenable to switching (e.g. structural, electronic, optical absorption, luminescence, magnetic), and an equally wide variety of external stimuli can be used to provide the switching action (e.g. changes in $\mathrm{pH}$, temperature, or electrochemical potential; the presence or absence of a metal ion; absorption of light).

Surface-bounded molecular switches, rotors and motors are fascinating since they can be exposed to external stimuli [4-6] and investigated by surface analysis methods [7-10]. Recent studies have shown that surface-bounded molecular switches and rotors can be used in experimental techniques such as scanning tunneling microscopy and spectroscopy (STM/STS) which also allow the measurement of molecular switching/rotor rates and energy barriers [11-14]. The need for monitoring the ordered assembly and manipulating its electronic property at the molecular level has made STM a widely employed and highly powerful technique for the investigation of molecular electronics at interfaces with submolecular resolution. In the late 1990s it was reported that propeller-shaped hexa-tert-butyl decacyclene molecules on a $\mathrm{Cu}$ (100) surface had an energy barrier for rotation below the thermal energy at room temperature and the rotation of the molecules could be manipulated by STM [15].

Azobenzene molecules, widely used as molecular switches, show reversible trans-cis isomerization. Upon irradiation by UV or visible light, the trans isomer which is thermodynamically more stable converts to a cis isomer. The cis-trans transition which has a smaller activation energy barrier than the trans-cis transition occurs thermally or through radiation at a lower energy than the trans-cis transition. Alemani et al illustrated the isomerization of azobenzene molecules by inducing an electric field using STM [16]. The trans-cis isomerization of the double bond, and the reversible switching, adsorption and manipulation of azobenzene molecules have been investigated using STM [17-24].

Acetylene [25] and cis-2-butene [26] molecules and their conformational changes have been investigated with the use of inelastic electron tunneling experiments. The movement and dynamics of chloromethyl- and dichloromethylsylil molecules on fused silica surfaces have been analyzed by STM [4, 5, 27].

Previously, we have reported the light-activated switching and structural rearrangements of self-assembled monolayers (SAMs) of anthracene compounds bound to a gold surface [28]. Anthracene compounds undergo reversible dimerization reactions when irradiated by light of an appropriate wavelength, a property that has been utilized in the preparation of molecular switches in a variety of configurations. In addition to experimental observations, significant theoretical research efforts have also been directed towards understanding of switching dynamics and molecule-tip interaction [29-33]. We also described temperature-responsive SAMs of a new OEG derivative that can be used to control the affinity binding of biological molecules on surfaces in a temperature-dependent manner. The OEG-derived
SAMs have been shown to reversibly switch the wettability of the surface [34].

Guirado et al explored the electrochemical behavior of simple photochromic dithienylethylenes with either a perfluoro or a perhydro cyclopentene ring. In addition to the experimental study, density functional theory (DFT) calculations provided information on the total energy, the geometry, and the electronic structures of several representative compounds [35]. Jang et al reported the structure and properties of SAMs of bistable [2] rotaxane molecules on Au (111) surfaces as a function of surface coverage using atomistic molecular dynamics studies together with DFT calculations, and they also reported several experiments that justified their predictions [36]. Zheng at al demonstrated experimentally a molecular-level active-plasmonic device that can be operated by switching the extinction properties of a bistable [2] rotaxane and provided a time-dependent DFTbased model [37]. Landge et al reported a computational analysis of the switching between two isomers of a phenylcontaining naphthyl-based system using DFT and natural bond orbital wave function analyses. They showed that the favorable isomerization mechanism in these hydrogen-bonded systems is hydrazine-azo tautomerization followed by rotation around a $\mathrm{C}-\mathrm{N}$ single bond [38]. In another study, by performing DFT calculations, Durgun et al showed that the molecular rings formed by connecting well-known photoswitchable molecules with suitable linker agents can simultaneously increase the stored energy in these systems through a switching mechanism [39].

In this work, we present data showing that (E)-4-((4(phenylethynyl) benzylidene) amino) benzenethiol (EPBB) molecules, a newly designed Schiff base, form monolayer structures on Au (111) surfaces by solution phase deposition. STM/STS and surface-enhanced Raman scattering (SERS) experiments were used to probe the conformational change and/or switching of the EPBB molecules with increasing tunneling current. The interpretation of our results was further supported by DFT calculations.

\section{Experimental section}

\subsection{Materials}

$\mathrm{Au}$ (111) films on mica were purchased from Phasis, Switzerland. The STM tip was prepared from Pt/Ir wire cut under ambient conditions. All chemicals were used as received: chloroform (>99\%, Merck), ethanol (>99\%, Sigma-Aldrich), phenylacetylene $(>98 \%$, Sigma), ethynyltrimethylsilane (>98\%, Sigma), 4-bromobenzaldehyde (>99\%, Sigma), 4-aminobenzenethiol (>98\%, Sigma), bis(triphenylphosphine)palladium(II) dichloride (>98\%, Sigma-Aldrich), copper(I) iodide (>98\%, Sigma), hexane (>98\%, SigmaAldrich), and diethyl ether ( $\geqslant 99 \%$, Sigma-Aldrich). Dichloromethane $\left(\mathrm{CH}_{2} \mathrm{Cl}_{2}\right)$ was dried over calcium hydride. THF was freshly prepared over $\mathrm{Na}$ metal in the presence of benzophenone as an indicator. Column chromatography was conducted with a silica gel 200-425 mesh. The structure of 


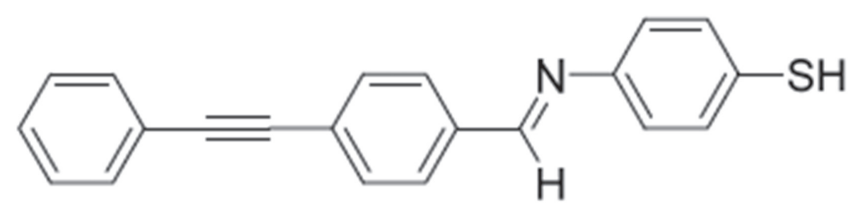

Figure 1. The EPBB molecule used in this work.

the prepared compounds was confirmed using ${ }^{1} \mathrm{H}$ NMR (500 MHz) and ${ }^{13} \mathrm{C}$ NMR (125 MHz).

The detailed procedures and characterization data for the prepared compounds are given in supporting information. Briefly, during the construction of novel Schiff base molecular switch EPBB, palladium/copper-catalyzed Sonogashira coupling between alkynylaryl and aryl halide which has an aldehyde moiety was conducted. Afterwards dehydration reaction between aldehyde and aminothiol yielded the product EPBB. The synthesis of the EPBB molecule depicted in figure 1 is described in the supporting information.

\subsection{Formation of SAMs}

SAMs of EPBB were prepared on Au (111) by liquid-solid phase assembly. Monolayer structures were prepared by immersing the gold substrate in $2-3 \mathrm{ml}$ solutions of the compound dissolved in dichloromethane or chloroform $(0.1 \mathrm{mM}-1 \mathrm{mM})$ under oxygen-free conditions in a container previously cleaned in a bath of $\mathrm{KOH} / \mathrm{H}_{2} \mathrm{O}_{2} / 2$-propanol (15/ $15 / 1$ ) (CAUTION). The container was sealed and placed in the dark at room temperature for $24 \mathrm{~h}$. Additionally, a sample was prepared at $55^{\circ} \mathrm{C}$ for $24 \mathrm{~h}$. The substrates were then removed from the solution and washed thoroughly with solvent and dried using a stream of $\mathrm{N}_{2}$ gas. Each sample was imaged using STM. X-ray photoelectron spectroscopy (XPS), ellipsometry and contact angle experiments were performed on samples prepared at room temperature.

\section{3. $X P S$}

Photoemission spectra were recorded with a Thermo Scientific K-Alpha XPS system furnished with a monochromatized Al-K $\alpha$ X-ray source. Freshly prepared samples of molecular structures on $\mathrm{Au}$ (111) were attached to the sample holder with conductive carbon adhesive tape from the back-side. A flood gun, a source of low-energy charged particles (é, $\mathrm{Ar}^{+}$), was used for charge compensation during the measurements. The base pressure of the vacuum chamber rose from $2.0 \times 10^{-9}$ mbar to $2.0 \times 10^{-7}$ mbar during the measurements. The X-ray spot size was approximately $400 \mu \mathrm{m}$. The spectra were recorded with a pass energy of $50 \mathrm{eV}$, which corresponds to a spectral resolution of ca. $0.5 \mathrm{eV}$. The spectra were referenced to the $\mathrm{C} 1 \mathrm{~s}$ binding energy at $284.8 \mathrm{eV}$. Spectral fitting was performed with Advantage v5 software.

\subsection{Ellipsometry and contact angle}

Ellipsometry measurements were performed using J.A. Woollam spectroscopic ellipsometer (M-2000) with a $2 \mathrm{~mm}$ laser diameter. Spectroscopic data were obtained over a spectral range of 245 to $1000 \mathrm{~nm}$ with an incidence angle of $65^{\circ}$. A Cauchy layer model was used to fit the data set. Contact angle measurements were performed using an Attension Theta optical tensiometer by Biolin Scientific, after dispensing droplets of DI water on the surface. The angle between the water droplet and surface was measured to determine the wettability of the samples.

\subsection{Computational methods}

The structural optimization and adsorption characteristics of EPBB molecules on $\mathrm{Au}$ (111) surfaces reported here are based on DFT calculations using a localized orbital basis and pseudopotentials to solve the Kohn-Sham equations as implemented in the SIESTA code [40]. The Perdew-BurkeErnzerhof [41] form of the generalized gradient approximation was adopted to describe the electron exchange and correlation. For simulating EPBB molecules on $\mathrm{Au}$ (111), calculations were performed using a two-layer gold substrate model and supercells. The plane-wave cutoff energy was set to $300 \mathrm{eV}$ in all calculations. The Brillouin zone was sampled using a $\Gamma$-centered $k$-point mesh and a Gaussian smearing of $0.01 \mathrm{eV}$ was used for total energy calculations. Structural relaxations were performed using a conjugate gradient method where the total energy and atomic forces were minimized. The convergence criterion for energy was set at $10^{-4} \mathrm{eV}$ between successive iteration steps, and the maximum force allowed on each atom was less than $10^{-4} \mathrm{eV} \AA^{-1}$. Since the studied structure had no source of magnetism, spinunpolarized calculations were performed in all cases.

\subsection{STM/STS}

STM images were acquired using a Nanosurf Easyscan system under ambient conditions. STM piezoelectric scanners were calibrated laterally, with graphite and $\mathrm{Au}$ (111), and vertically, using the height of the Au (111) steps (2.2 $⿱$ ) [4244]. The STM tip was prepared from a Pt/Ir (90\%/10\%) $0.25-0.2 \mathrm{~mm}$ wire cut under ambient conditions. All images were acquired in a constant-current mode. The typical imaging conditions were a bias voltage of $1 \mathrm{~V}$ and a tunneling current of $5 \mathrm{pA}$ to $1.5 \mathrm{nA}$. The images shown are raw data unless stated otherwise. The images were manipulated with Scanning Probe Image Processor software. Contrast-enhanced images were obtained by applying a correlation averaging procedure to analyze repeating molecular units and by applying a low-pass filter.

\subsection{SERS}

Raman spectrometer XploRA (Horiba) equipped with a confocal microscope was used. Raman signals were recorded in a spectral range of $600-2500 \mathrm{~cm}^{-1}$ using a $785 \mathrm{~nm}$ laser excitation, in combination with a $40 \mathrm{X}$ objective magnification $(\mathrm{NA}=0.65)$ of an Olympus BX41 transmission and reflection illumination microscope (Olympus, France) for the focusing and collection of Raman-scattered light. An EPBB molecule was dissolved in chloroform $(1 \mathrm{mM})$ and dropped onto a SERS plate (S. T. Japan Inc.). In order to investigate 


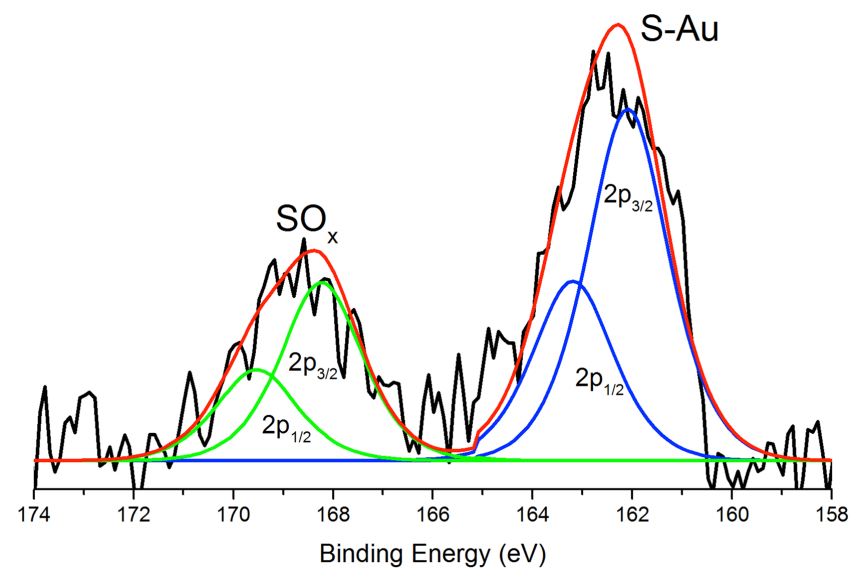

Figure 2. XPS spectra showing the $S 2 p$ region of the EPBB molecule.

the trans-cis transition, the substrate which was dropped onto the EPBB solution was irradiated by UV light at $365 \mathrm{~nm}$ and then the substrate was heated to $55^{\circ} \mathrm{C}$ to induce the transition from cis to trans [45]. For each sample, the Raman experiment was repeated five times to check the reproducibility of the measurement. Each spectrum was normalized using LabSpec software.

\section{Results and discussion}

\subsection{XPS}

The XPS spectrum of the sample is given in figure 2. The C 1s spectrum (see supporting information, S5) was deconvoluted into three peaks: a major carbon species at $284.8 \mathrm{eV}$ and two minor peaks at $287.5 \mathrm{eV}$ and $290.7 \mathrm{eV}$. The ratio of the intensities was 9.6: 1.4: 1.0, respectively. The major peak was assigned to the aromatic carbon species in the molecule. The peak at $287.5 \mathrm{eV}$ was the carbonyl groups while the peak at $290.7 \mathrm{eV}$ was most likely due to O(CO)O-type moieties from the impurities on the surface. The $\mathrm{N} 1 \mathrm{~s}$ region (see supporting information, S5) showed a broad peak, which could be deconvoluted into two peaks at $398.6 \mathrm{eV}$ and $401.0 \mathrm{eV}$ with a 1.7 to 1.0 ratio. While the stronger peak at lower binding energy corresponded to the nitrogen atoms within the molecule, the weaker peak at higher binding energy suggested an oxidized nitrogen species. The sulfur spectrum (figure 2) showed two $2 \mathrm{p}$ doublets at $161.8 \mathrm{eV}\left(2 \mathrm{p}_{3 / 2}\right)$ and $167.9 \mathrm{eV}$ $\left(2 \mathrm{p}_{3 / 2}\right)$. The first doublet can be assigned to sulfur linking the molecule to the gold substrate, while the latter indicates the presence of oxidized sulfur species. The $\mathrm{O} 1 \mathrm{~s}$ region showed a weak and broad peak at $531.7 \mathrm{eV}$. The presence of oxygen and oxidized nitrogen, sulfur and carbon species reveals a slight inclination towards oxidation. As seen in figure 2, the intensity of the $2 \mathrm{p}_{3 / 2}$ peak at $167.9 \mathrm{eV}$ for the oxidized sulfur $\left(\mathrm{SO}_{x}\right)$ species reached about half the intensity of the thiol sulfur. This is an expected result as the oxidation of sulfur proceeds with a lower activation barrier than that of carbon. The oxidized carbon species (see S5), on the other hand, have
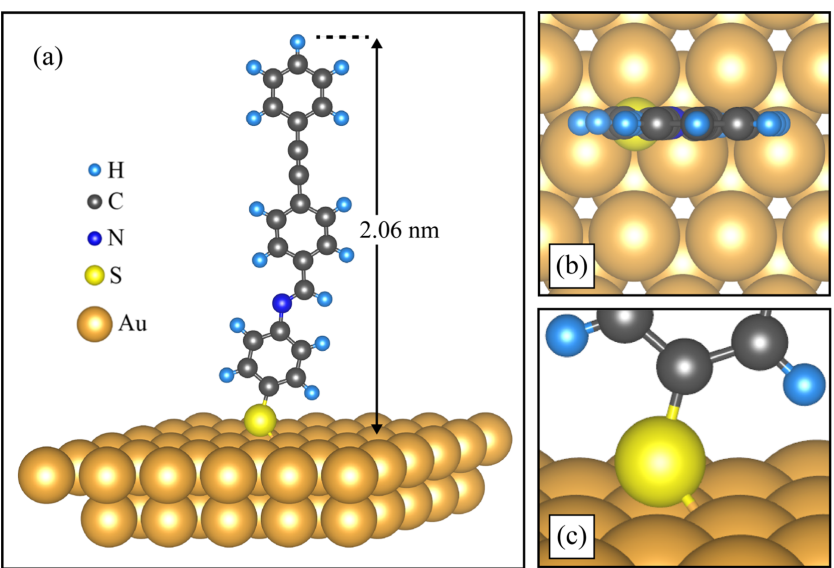

Figure 3. (a) Side view of optimized single EPBB molecule on Au (111) surface. (b) Top view and (c) tilted side view.

only $10 \%$ of the aliphatic carbon intensity, at $287.5 \mathrm{eV}$ and $290.5 \mathrm{eV}$ for the carbonyl and carboxyl moieties, respectively.

\subsection{Ellipsometry and contact angle}

The thickness of the deposited layer on gold was measured by ellipsometry. A thickness of $2.12 \pm 0.1 \mathrm{~nm}$ was obtained by using a refractive index of 1.44 for the organic layer, determined via a refractometer. This value was in good agreement with the theoretically calculated thickness of the EPBB molecules (figure 3) assembled through S-Au bonding on the surface with a $30^{\circ}$ angle. The measured thickness was slightly less than the thickness of $2.12 \pm 0.1 \mathrm{~nm}$ for an EPBB monolayer in a perpendicular orientation relative to the surface, suggesting that the molecules were not perfectly perpendicular with respect to the gold surface. Indeed there may be some areas that were not monolayer. These areas of defects may be due to unorganized SAMs that may appear from multilayer or $\mathrm{Au}$ (111) defects. However, the thickness measurement performed by ellipsometry showed that the sample was mainly composed of a SAM. A figure of the ellipsometry measurements showing the change of monolayer thickness is given in the supporting information (see figure S6). Water contact angles were measured on freshly prepared surfaces. An advancing angle of $70^{\circ}$ was recorded for the EPBB molecules on the Au (111) surface, while an angle of $64^{\circ}$ was recorded for the untreated gold substrate under the same experimental conditions.

Theoretically, we first calculated the structural properties and interaction characteristics of a single EPBB molecule with the $\mathrm{Au}$ (111) surface. Based on the given parameters in the experimental section, the single EPBB molecule was optimized initially in the vacuum, and the optimized EPBB molecule was then relaxed on the $\mathrm{Au}$ (111) surface. Our calculations showed that the atomic structure of a single isolated molecule does not change significantly after being adsorbed on a $\mathrm{Au}$ (111) surface (see figure 3). It was also seen that similar to its vacuum state each single EPBB molecule adsorbed on the Au surface was in trans conformation. It was calculated that a single EPBB molecule in Au (111) trans state 

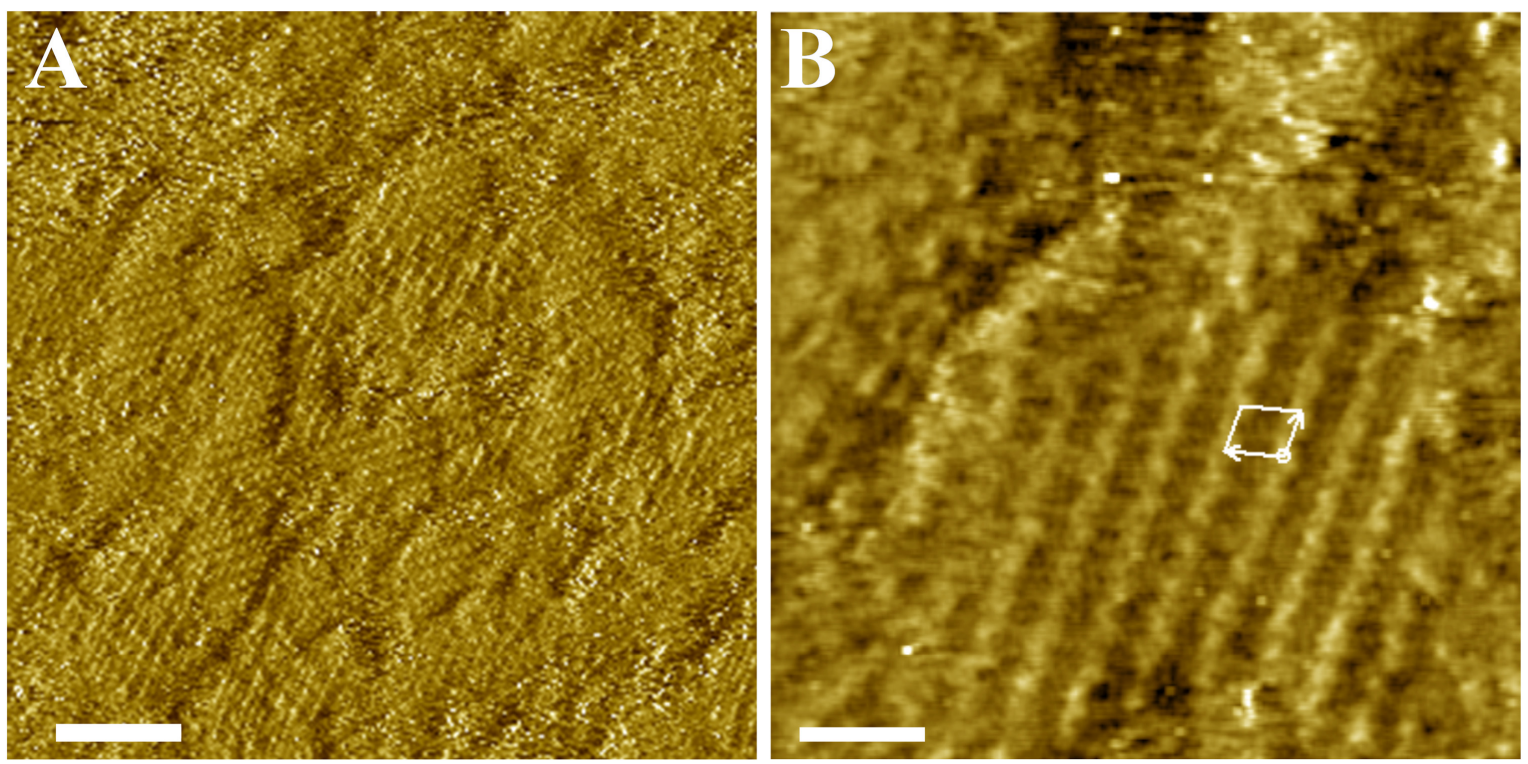

Figure 4. (A) STM image of a $\mathrm{Au}$ (111) surface after immersion in a chloroform solution of EPBB molecules (scale bar $=5.2 \mathrm{~nm})$. (B) Magnified view of an ordered region from image A (scale bar $=1.5 \mathrm{~nm})$

had $242 \mathrm{meV}$, more favorable than that in the cis state. In this form, the height of the molecule, which was measured as the vertical distance of the top-most $\mathrm{H}$ atom to the gold surface, was $2.06 \mathrm{~nm}$. The $\mathrm{S}$ atom at the bottom localized near the center of the triangular interatomic site on the Au (111) surface (figure 3(b)). Yet, the $S$ atom preferred to be slightly closer to one of the Au atoms with a bond length of $2.49 \AA$ (the distances to the other two Au atoms were $2.50 \AA$ and $2.53 \AA$ ). In addition, the $\mathrm{C}-\mathrm{S}-\mathrm{Au}$ angle of the binding site was about $126^{\circ}$. Our total energy calculations also reveal that the binding energy $\left(E_{\text {bind }}=E_{\mathrm{EPBB}}+E_{\text {Surface }}-E_{\text {Total }}\right)$ of a single EPBB molecule to the Au (111) surface was $346 \mathrm{meV}$. Therefore, one can expect the stable binding of EPBB molecules on the Au surface at room temperature. Moreover, when the SAM structures were formed, the binding energy per molecule was enhanced due to intermolecular interaction.

\subsection{Preparation and characterization of SAMs}

The structures of the SAMs of EPBB molecular films were studied at atomic resolution using STM. A representative large-area STM image is shown in figure 4(A). This image contains many short-range islands with average sizes of 5 to $15 \mathrm{~nm}$ on $\mathrm{Au}$ (111). According to the images taken from 50 different areas of the same sample, approximately $80 \%$ of the surface was found to be occupied with these islands and the rest was generally covered by unorganized molecules.

The organization or assembly of EPBB molecules as shown in figure 4(B) demonstrated that the molecules were ordered in parallel rows and separated by $0.78 \mathrm{~nm}$ from each other. The domains yielded a nearly-oblique lattice with parameters $a=0.68 \pm 0.1 \mathrm{~nm}, b=0.8 \pm 0.1 \mathrm{~nm}$ and $\alpha=101^{\circ}$ as determined from the STM image in figure 4(B).

The template in the organization of EPBB molecules on the $\mathrm{Au}$ surface had the 2D triangular lattice symmetry of the $\mathrm{Au}$ (111) substrate. Considering the strong binding of the $\mathrm{S}$ atoms to the Au surface one can obtain various possible stable arrangements of the EPBB molecules. Among these we studied five different lattice types for the SAM: triangular, rectangular, and three oblique lattices (the details of the calculations are given in the supporting information, figure S8). The molecular lattice compatible with the experimental STM images was identified as an oblique lattice with parameters $a=0.58 \mathrm{~nm}, b=0.77 \mathrm{~nm}$, and $\alpha=100.9^{\circ}$ (see figure 5).

Here it is worthwhile to note that the internal atomic structure of an EPBB molecule changes considerably when trans isomers form a periodic SAM structure as shown in figure 5. The $\pi-\pi$ interaction of the phenyl groups and the interaction between the hydrogen atoms on the phenyl rings make the lower and the upper benzene rings change their orientation. In figure 5 the simulated SAM lattices and the atomic structure of an EPBB molecule when arranged in a close-packed order on the surface are also presented. The upper and lower panels refer to the trans and cis conformations of the EPBB molecules, respectively. These were identified as the two cases corresponding to the distinct images observed in the STM scans (see figure 7). Both arrangements shared the same oblique lattice; however when the molecules transformed to the cis form by bending at the $\mathrm{N}$ junction, each molecule started to produce a double spot in the STM images. The bending mechanism also lowered the height of the SAM from $2.03 \mathrm{~nm}$ to $1.26 \mathrm{~nm}$ from the line profile on the images (not shown).

The STM experiments were conducted at 20 to 50 different regions of the sample. Thus cis formation took place in the entire sample when the sample came into contact with the STM tip. The average thickness in the cis position was therefore comparable with the value obtained in the ellipsometry measurements. The structural transformation of the SAM while being scanned at high-current mode was 


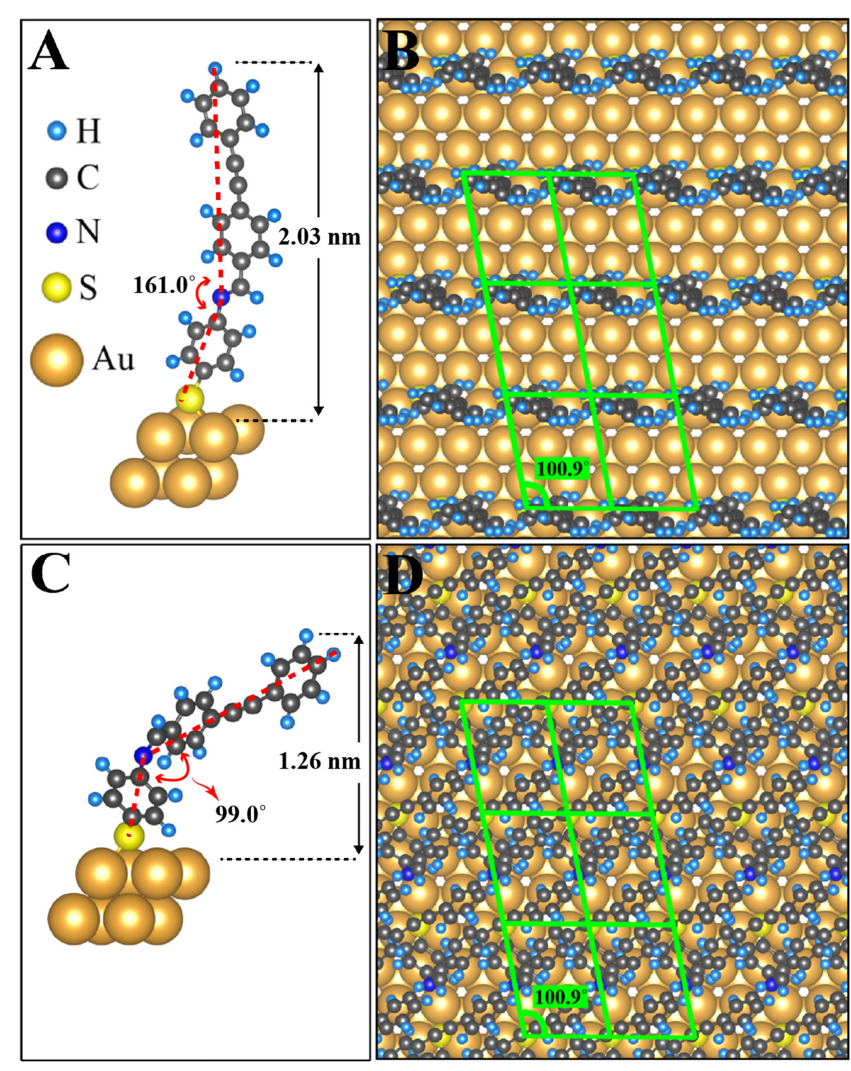

Figure 5. Simulated SAM lattices and the atomic structure of an EPBB molecule when arranged in a close-packed order on the surface. The upper (A and $\mathrm{B})$ and lower $(\mathrm{C}$ and $\mathrm{D})$ panels refer to the trans and cis conformations of the EPBB molecules, respectively.

attributed to the strong interaction of the STM tip with the tail groups of the EPBB molecules adsorbed on Au (111). In order to estimate the strength of this interaction, we calculated the potential profile as a function of the tip-molecule distance.

As shown in figure 6(A), we simulated the STM tip with a $\mathrm{Pt}_{10}$ cluster. From the interaction profile of the tip (see figure 6(B)) it is clear that the Pt tip interacted strongly with the EPBB molecules. The strength of the interaction was found to be $350 \mathrm{meV}$ which is strong enough to induce morphology changes in the SAMs but not larger than the binding energy of a single molecule in the SAMs. Therefore, this interaction energy cannot lead to detachment of the molecules from the $\mathrm{Au}$ (111) surface. This strong interaction at reduced distances seemed to trigger the trans-to-cis transformation of the molecules in a regular manner, producing the apparent reorganization of the SAMs in the STM scan (figure $7(\mathrm{H})$ ).

In the next experiment on the switching mechanism, we applied a tunneling current to the domains in which the EPBB molecules were in the trans configuration (figures 7(A) and (B)). The enlarged STM image (figure 7(B)) with the same tunneling current $(5 \mathrm{pA})$ and bias voltage $(1 \mathrm{~V})$ condition showed no changes yet in the morphology of the SAM. While increasing the tunneling current from $50 \mathrm{pA}(7 \mathrm{C})$, to $400 \mathrm{pA}$ (7D), $600 \mathrm{pA}(7 \mathrm{E}), 800 \mathrm{pA}(7 \mathrm{~F}), 1 \mathrm{nA}(7 \mathrm{G})$ and $1.2 \mathrm{nA}(7 \mathrm{H})$, we observed that the STM tip could change the EPBB
A

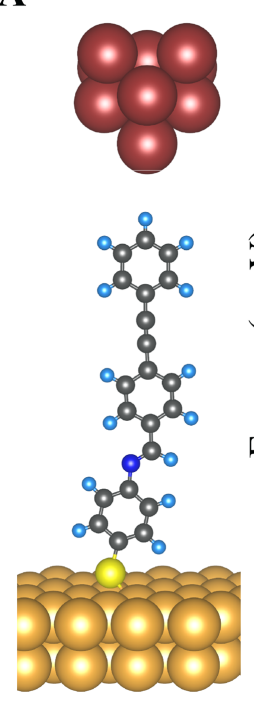

B

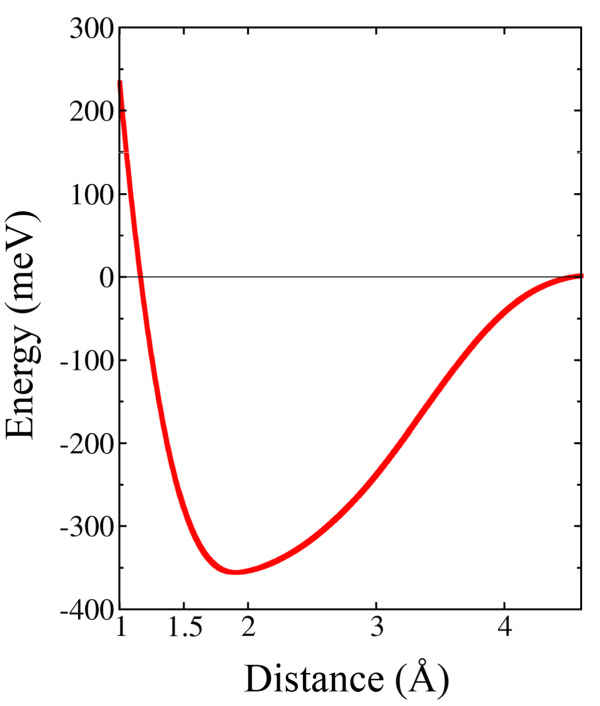

Figure 6. (A) STM tip $\left(\mathrm{Pt}_{10}\right)$ placed above an isolated EPBB molecule bound to the Au surface. (B) Interaction energy between the molecule and the tip.

monolayer significantly when the current was increased to $1.2 \mathrm{nA}$. Here, one can clearly observe that for lower tunneling currents there were only slight changes in the rows of the EPBB monolayer (figures $7(\mathrm{C})-(\mathrm{F})$ ). But after the current reached 1-1.2 nA the morphology of the monolayer changed significantly (compare figures $7(\mathrm{G})$ and $(\mathrm{H})$ ). This may be due to the molecular architecture tending to switch from trans to cis form. As one can see, all the molecules in the island (figure $7(\mathrm{H})$ ) changed their appearance, showing a lower height $(1.2 \AA$ from the ellipsometry measurement) with respect to the one in figure $7(B)$. The image in figure $7(\mathrm{H})$ indicated that individual EPBB molecules in the monolayer were seen with a higher resolution, and the number of rows was doubled, due to the rotation and switching of the molecules induced by the effect of the tunneling current. In this current range there was not any tip effect on the images. Also, the conformational change was reproducible as the experiment was performed on at least 20 to 30 different areas of the sample. It is clear that the ability to change the conformation by tuning the tunneling current allowed us to control the process. The transition was reversible only with external stimuli such as heating. A temperature of $55{ }^{\circ} \mathrm{C}$ turned the molecules from cis to trans form. The observed conformational change was unique because of two main reasons: (i) The EPBB molecule had trans and cis isomers and their energies were comparable to each other. Therefore, the transition that could not be observed for other molecules such as thiophenol (see figure S7, controlled experiments) took place for the assembly of a special EPBB molecule. (ii) The attractive interaction between the uppermost part of the EPBB molecule and the apex of the STM tip was essential. For noninteracting and repulsive-interacting cases one could not have structural transformation from one well-ordered lattice type to another. To sum up, the type of molecule and the type of interaction with the STM tip were crucial for the mechanism 


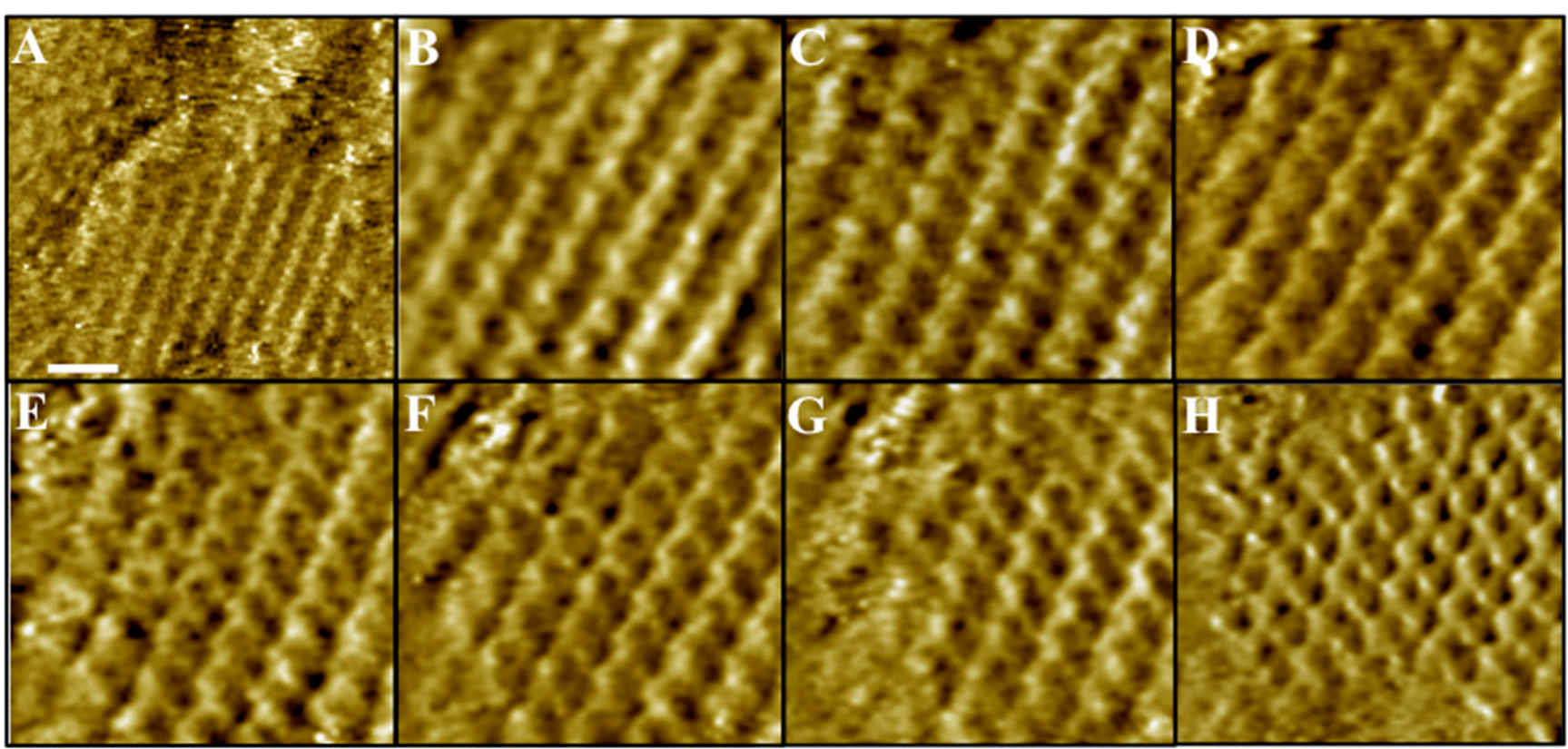

Figure 7. STM images of ordered assemblies of EPBB. The STM images were recorded under constant-current mode with tunneling current varying between $5 \mathrm{pA}$ and $1.2 \mathrm{nA}$ and a $1 \mathrm{~V}$ bias voltage. Images (A) and (B) $5 \mathrm{pA}$, (C) $50 \mathrm{pA}$, (D) $400 \mathrm{pA}$, (E) $600 \mathrm{pA},(\mathrm{F}) 800 \mathrm{pA},(\mathrm{G}) 1 \mathrm{nA}$ and $(\mathrm{H}) 1.2 \mathrm{nA}$. The scale bar in $(\mathrm{A})$ is $1.5 \mathrm{~nm}$ and for $(\mathrm{B})-(\mathrm{H})$ it is $0.8 \mathrm{~nm}$.

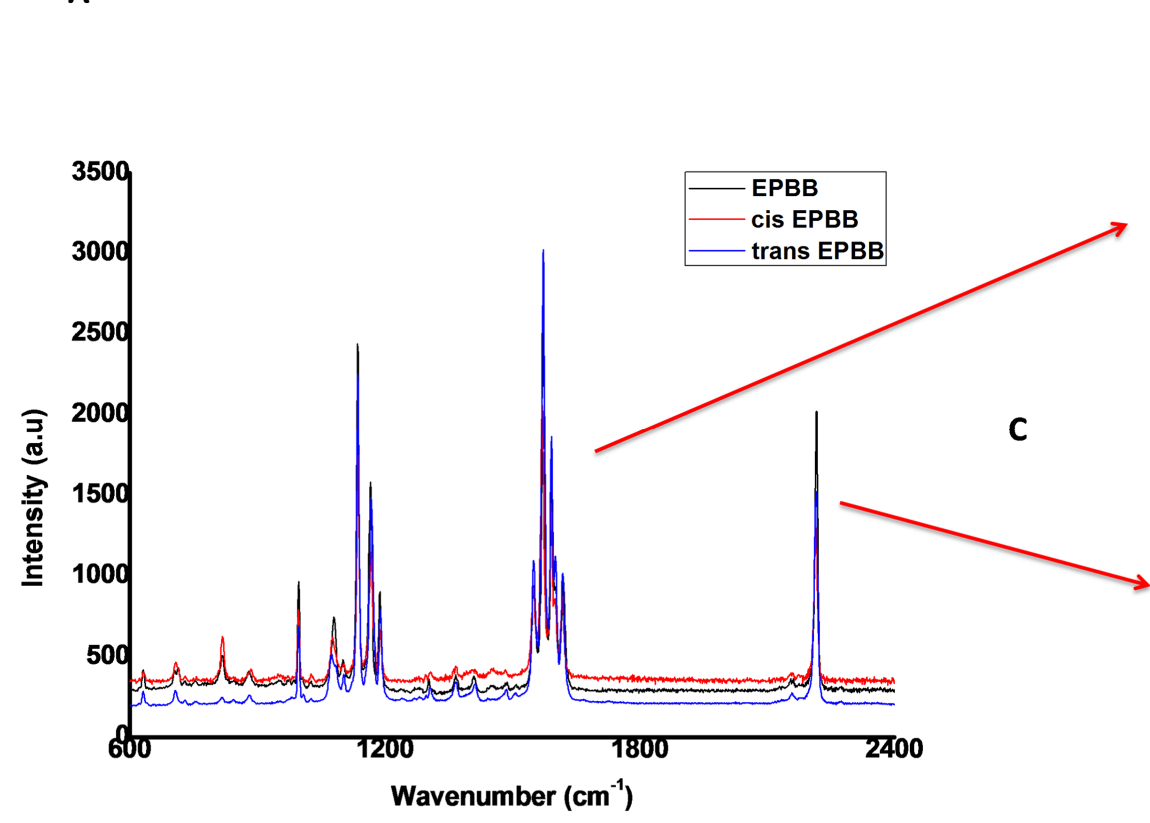

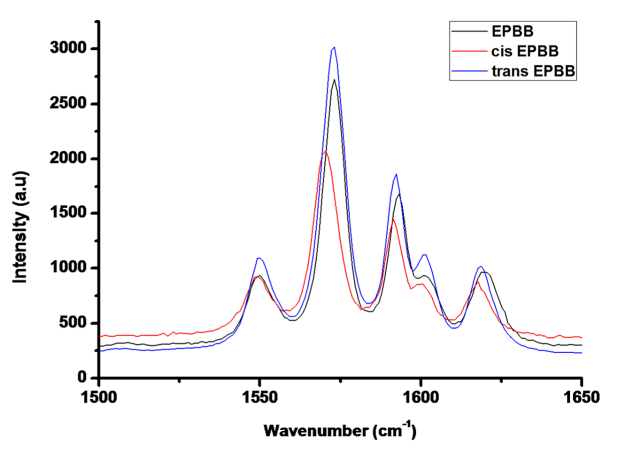

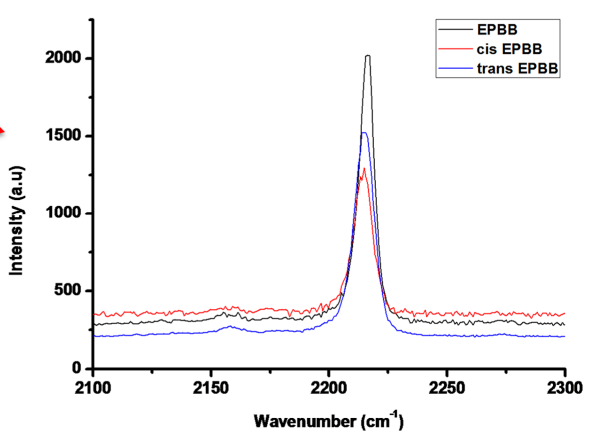

Figure 8. (A) SERS spectra of EPBB SAMs. The black spectra illustrate the EPBB molecules before UV irradiation or trans mode, the red spectra demonstrate the cis form of the SAMs after UV irradiation and the blue spectra indicate the cis-to-trans isomerization after heating to $55^{\circ} \mathrm{C}$. (B) Enlarged spectrum of $1500-1650 \mathrm{~cm}^{-1}$, and (C) enlarged spectrum of $2200-2230 \mathrm{~cm}^{-1}$.

of transformation. STM was conducted as well to observe the reversibility of the molecular transition from cis to trans isomer. Unfortunately, increasing the temperature to $55^{\circ} \mathrm{C}$, which was essential for driving the cis-to-trans transition, disturbed the quality of STM images.

The SERS experiments were conducted to verify the transition of the EPBB molecules from trans to cis and its reversibility. Figure 8 shows the Raman spectrum of EPBB obtained with $785 \mathrm{~nm}$ laser. Figure 8(A) depicts the Raman spectra of the trans form of the EPBB molecules (black spectrum). After $365 \mathrm{~nm}$ UV irradiation the EPBB molecules switched into cis form (red spectrum). Upon the ceasing of UV irradiation and heating the sample up to $55^{\circ} \mathrm{C}$, the EPBB molecules switched to the initial trans form (blue spectrum). 


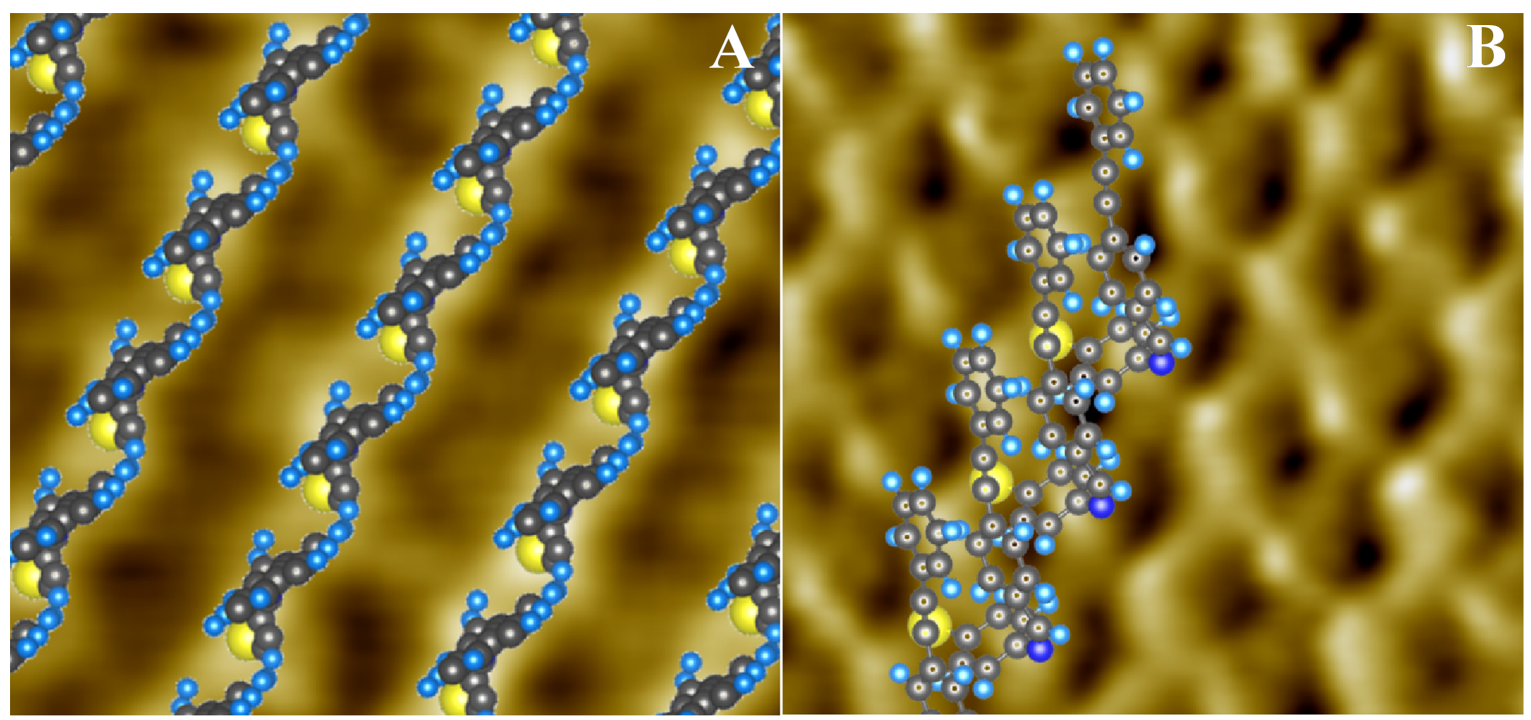

Figure 9. (A) STM image of figure 7(B) together with the calculated lattice of trans-EPBB molecules. (B) STM image of figure 7(G) together with the calculated lattice of cis-EPBB molecules. The lattice of $\mathrm{S}$ atoms bound to the molecules was the same for both cases. Image sizes $=2.5 \times 2.5 \mathrm{~nm}$.

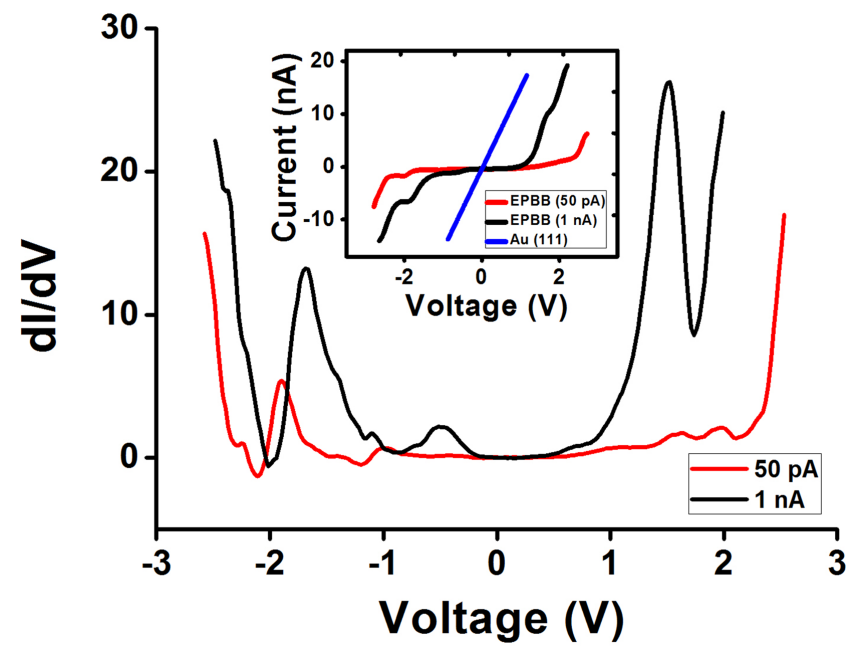

Figure 10. $\mathrm{d} I / \mathrm{d} V$ characteristics of the EPBB molecule and of a $\mathrm{Au}$ substrate on mica. The inset shows the current-voltage characteristics of the individual molecules of EPBB compared to those from a bare Au surface.

EPBB interacts with solid interfaces via the thiol functional group forming metal-S-C bonds. The peak at $665 \mathrm{~cm}^{-1}$ corresponded to the C-S stretch [46]. In the spectra (figure $8(\mathrm{~B})$ ), we mainly focused on a specific range between $1540 \mathrm{~cm}^{-1}$ and $1630 \mathrm{~cm}^{-1}$ to identify the cis-trans transition. The peaks at $1573 \mathrm{~cm}^{-1}, 1593 \mathrm{~cm}^{-1}$ and $1619 \mathrm{~cm}^{-1}$, respectively, in the black spectrum arose from the $\mathrm{C}-\mathrm{C}$ stretch and $\mathrm{C}=\mathrm{C}$ stretch of aromatic rings and the $\mathrm{C}=\mathrm{N}$ stretch of the molecule in trans form. After irradiation by UV light, these peaks shifted to lower wavelengths indicating the formation of a cis isomer (red spectrum) (figure 8(B)) [47]. The strong $\mathrm{C} \equiv \mathrm{C}$ stretch of the ethynyl group was observed at $2216 \mathrm{~cm}^{-1}$ as shown in figure $8(\mathrm{C})$ and this stretching mode also shifted due to the rotation of the bond upon cis isomerization. In the range between $700 \mathrm{~cm}^{-1}$ and $1300 \mathrm{~cm}^{-1}$, these peaks showed
C-C stretch, C-C, C-H bending and ring breathing modes (figure 8(A)) [48, 49].

The observed changes in the pattern of the SAMs could be explained by considering the trans and cis conformations of the EPBB molecules. As stated before, for an isolated EPBB molecule, the energy of the trans form was found to be $242 \mathrm{meV}$ lower than that of the cis form. When these molecules were self-assembled on the Au surface, they preserved the trans form while being imaged under relatively low current. As seen in figure $7(\mathrm{H})$ the pattern of the SAMs noticeably changed at the high-current operation mode of the STM. In the new pattern, the rows of bright spots seemed to double while preserving the symmetry and lattice parameters of the oblique lattice. This indicated a conformational change of the adsorbed molecules. Regarding the strong interaction potential $(\sim 350 \mathrm{meV})$ between the STM tip and the EPBB molecules, an STM-tip-induced trans-to-cis transformation might be a reasonable mechanism for the observed changes. Moreover, our calculations revealed that unlike the case of isolated molecules, the cis form of the EPBB was energetically more favorable than the trans form by $786 \mathrm{meV}$ per molecule when they formed a periodic SAM structure. Such a conformational change of the molecules was also consistent with the thickness measurement of the SAM. The measured height of the monolayer decreased to $1.2 \AA$ upon transition to the cis form.

As shown in figure 9, the superimposed simulated atomic structures of the SAMs and their STM images were in good agreement. The transition from trans to cis form was achieved through a bending of the molecule around the $\mathrm{N}$ atom. The emergence of additional rows in the STM image was attributed to the increased contribution of the lower phenyl groups of the molecules to the tunneling current.

Finally, we investigated the electronic behavior of the EPBB SAMs by establishing their current/voltage $\mathrm{d} I / \mathrm{d} V$ and 
$I / V$ characteristics via STS (figure 10). The STS measurements were performed at a fixed tip-sample distance with the feedback loop switched off. Each $I / V$ curve was averaged over at least 20 successive voltage sweeps. The time required for a complete spectroscopy measurement was below $0.1 \mathrm{~s}$; therefore any influence of lateral drift was neglected. The inset curves in figure 10 show the $I / V$ characteristics of bare $\mathrm{Au}$ (111), and the EPBB monolayer at $50 \mathrm{pA}$ (red) and $1.2 \mathrm{nA}$ (black), respectively. Both of the $I / V$ curves of EPBB in homoassembly showed semiconducting behavior, evident when compared with the bare $\mathrm{Au}$ (111) surface. On the basis of the $\mathrm{d} I / \mathrm{d} V$ characteristic, these molecules had an average energy gap of $2.5 \mathrm{eV}(50 \mathrm{pA})$ and $2.0 \mathrm{eV}(1 \mathrm{nA})$, respectively. The emergence of the peak at $0.5 \mathrm{eV}$ in the $\mathrm{d} I / \mathrm{d} V$ curve can be attributed to the presence of defect states that stemmed from unorganized EPBB molecules.

The density of states for both the trans and cis conformation of the individual molecules is presented in figure S9 (see supporting information). It appeared that when the transition took place from trans to cis the HOMO-LUMO gap reduced from $2.1 \mathrm{eV}$ to $1.7 \mathrm{eV}$ (19\% reduction). This was in good qualitative agreement with the $\mathrm{d} I / \mathrm{d} V$ measurements where the measured gap reduced from $2.5 \mathrm{eV}$ to $2.1 \mathrm{eV}(20 \%$ reduction).

\section{Conclusions}

The exposure of $\mathrm{Au}$ (111) surfaces to EPBB in basic chloroform or dichloromethane solution resulted in the formation of a short-range order and long-term stable monolayer. STM data showed that a chemisorption process took place (evidenced by etch pits) and that molecular-sized features were evident on the surface. The binding of EPBB onto gold through a $\mathrm{S}-\mathrm{Au}$ bond was determined by XPS. Furthermore, DFT calculations and STM/STS experiments were used to probe the conformational change or switching of EPBB molecules with increasing tunneling current. The SERS spectra clearly show the transition of EPBB molecules from trans to cis and its reversibility. We have provided evidence that those conformational changes were trans-cis isomer transitions.

\section{Acknowledgments}

The authors acknowledge financial support from TUBITAK (PROJECT NO: 112T507). This work was also supported by the Flemish Science Foundation (FWO-Vl). Computational resources were provided by TUBITAK ULAKBIM, High Performance and Grid Computing Center (TR-Grid-Infrastructure). HS is supported by an FWO Pegasus Long Marie Curie Fellowship.

\section{References}

[1] Shirai Y, Morin J F, Sasaki T, Guerrero J M and Tour J M 2006 Recent progress on nanovehicles Chem. Soc. Rev. 35 $1043-55$

[2] Tour J M 2000 Molecular electronics synthesis and testing of components Acc. Chem. Res. 33 791-804

[3] Collier C P, Wong E W, Belohradsky M, Raymo F M, Stoddart J F, Kuekes P J, Williams R S and Heath J R 1999 Electronically configurable molecular-based logic gates Science 285 391-4

[4] van Delden R A, ter Wiel M K J, Pollard M M, Vicario J, Koumura N and Feringa B L 2005 Unidirectional molecular motor on a gold surface Nature 437 1337-40

[5] London G, Carroll G T, Landaluce T F, Pollard M M, Rudolf P and Feringa B L 2009 Light-driven altitudinal molecular motors on surfaces Chem. Commun. 1712-14

[6] Comstock M J et al 2007 Reversible photomechanical switching of individual engineered molecules at a surface Phys. Rev. Lett. 99038301

[7] Henningsen N, Franke K J, Torrente I F, Sehulze G, Priewisch B, Ruck-Braun K, Dokic J, Klamroth T, Saalfrank P and Pascual J I 2007 Inducing the rotation of a single phenyl ring with tunneling electrons J. Phys. Chem. C 111 14843-8

[8] Stipe B C, Rezaei M A and Ho W 1998 Inducing and viewing the rotational motion of a single molecule Science 279 1907-9

[9] Manzano C, Soe W H, Wong H S, Ample F, Gourdon A, Chandrasekhar N and Joachim C 2009 Step-by-step rotation of a molecule-gear mounted on an atomic-scale axis Nat. Mater. 8 576-9

[10] Liljeroth P, Repp J and Meyer G 2007 Current-induced hydrogen tautomerization and conductance switching of naphthalocyanine molecules Science 317 1203-6

[11] Stohr M, Wagner T, Gabriel M, Weyers B and Moller R 2001 Direct observation of a hindered eccentric rotation of an individual molecule, $\mathrm{Cu}$-phthalocyanine on $\mathrm{C}_{60}$ Phys. Rev. $\mathrm{B}$ $\mathbf{6 5} 33404$

[12] Wahl M, Stohr M, Spillmann H, Jung T A and Gade L H 2007 Rotation-libration in a hierarchic supramolecular rotor-stator system: arrhenius activation and retardation by local interaction Chem. Commun. 1349-51

[13] Wintjes N, Bonifazi D, Cheng F Y, Kiebele A, Stohr M, Jung T A, Spillmann H and Diederich F 2007 A supramolecular multiposition rotary device Angew. Chem. Int. Ed. 46 4089-92

[14] Weigelt S, Busse C, Petersen L, Rauls E, Hammer B, Gothelf K V, Besenbacher F and Linderoth T R 2006 Chiral switching by spontaneous conformational change in adsorbed organicmolecules Nat. Mater. 5 112-17

[15] Gimzewski J K, Joachim C, Schlittler R R, Langlais V, Tang $\mathrm{H}$ and Johannsen I 1998 Rotation of a single molecule within a supramolecular bearing Science 281 531-3

[16] Alemani M, Peters M V, Hecht S, Rieder K-H, Moresco F and Grill L 2006 Electric field-induced isomerization of azobenzene by STM J. Am. Chem. Soc. 12814446

[17] Rau H 2003 Photochromism: Molecules and Systems ed H Dürr and H Bouas-Laurent (Amsterdam: Elsevier) p 165

[18] Fanghänel D, Timpe G and Orthman V 1990 Organic Photochromes ed A V El'tsov (New York: Consultants Bureau) p 105

[19] Nägele T, Hoche R, Zinth W and Wachtveitl J 1997 Femtosecond photoisomerization of cis-azobenzene Chem. Phys. Lett. 272 489-95

[20] Ishikawa T, Noro T and Shoda T 2001 Theoretical study on the photoisomerization of azobenzene J. Chem. Phys. 115 7503-12 
[21] Hugel T, Holland N B, Cattani A, Moroder L, Seitz M and Gaub H 2002 Single-molecule optomechanical cycle Science 296 1103-6

[22] Kirakosian A, Comstock M J, Cho J and Crommie M F 2005 Molecular commensurability with a surface reconstruction: STM study of azobenzene on Au (111) Phys. Rev. B 71 113409

[23] Comstock M J, Cho J, Kirakosian A and Crommie M F 2005 Manipulation of azobenzene molecules on $\mathrm{Au}(111)$ using scanning tunneling microscopy Phys. Rev. B 72153414

[24] Miwa J A, Weigelt S, Gersen H, Besenbacher F, Rosei F and Linderoth T 2006 Azobenzene on $\mathrm{Cu}(110)$ : adsorption site dependent diffusion J. Am. Chem. Soc. 128 3164-5

[25] Dunphy J C, Rose M, Behler S, Ogletree D F, Salmeron M and Sautet P 1998 Acetylene structure and dynamics on Pd(111) Phys. Rev. B 57 R12705-8

[26] Sainoo Y, Kim Y, Komeda T, Kawai M and Shigekawa H 2003 Observation of Cis-2-butene molecule on Pd(110) by cryogenic STM: site determination using tunneling-currentinduced rotation Surf. Sci. 536 L403-7

[27] Clarke L I, Horinek D, Kottas G S, Varaksa N, Magnera T F, Hinderer T P, Horansky R D, Michl J and Price J C 2002 The dielectric response of chloromethylsilyl and dichloromethylsilyl dipolar rotors on fused silica surfaces Nanotechnology 13 533-40

[28] Zareie H M, Barber J and McDonagh A M 2006 Structural changes in self-assembled monolayers initiated by ultraviolet light $J$. Phys. Chem. B 110 15951-4

[29] Horansky R D, Clarke L I, Winston E B and Price J C 2006 Dipolar rotor-rotor interactions in a difluorobenzene molecular rotor crystal Phys. Rev. B 74054306

[30] Gould S L, Tranchemontagne D, Yaghi O M and Garcia-Garibay M A 2008 Amphidynamic character of crystalline MOF-5: rotational dynamics of terephthalate phenylenes in a free-volume, sterically unhindered environment $J$. Am. Chem. Soc. 130 3246-7

[31] Winston E B, Lowell P J, Vacek J, Chocholousova J, Michl J and Price J C 2008 Dipolar Molecular rotors in the metal-organic framework crystal IRMOF-2 Phys. Chem. Chem. Phys. 10 5188-91

[32] Tierney H L, Calderon C E, Baber A E, Charles E, Sykes H and Wang F 2010 Understanding the rotational mechanism of a single molecule: STM and DFT investigations of dimethyl sulfide molecular rotors on $\mathrm{Au}(111)$ J. Phys. Chem. C 114 3152-5

[33] Yan S, Ding Z, Xie N, Gong H, Sun Q, Guo Y, Shan X, Meng S and Lu X 2012 Turning on and off the rotational oscillation of a single porphine molecule by molecular charge state ACS Nano 6 4132-6

[34] Zareie H M, Boyer C, Bulmus V, Nateghi E and Davis T P 2008 Temperature-responsive self-assembled monolayers of oligo(ethylene glycol): control of biomolecular recognition ACS Nano 2 757-65
[35] Guirado G, Coudret C, Hliwa M and Launay J P 2005 Understanding electrochromic processes initiated by dithienylcyclopentene cation-radicals J. Phys. Chem. B 109 17445-59

[36] Jang S S et al 2005 Structures and properties of self-assembled monolayers of bistable [2]Rotaxanes on Au (111) surfaces from molecular dynamics simulations validated with experiment J. Am. Chem. Soc. 127 1563-75

[37] Zheng Y B, Yang Y-W, Jensen L, Fang L, Juluri B K, Flood A H, Weiss P S, Stoddart J F and Huang T J 2009 Active molecular plasmonics: controlling plasmon resonances with molecular switches Nano Lett. 9 819-25

[38] Landge S M, Tkatchouk E, Benítez D, Lanfranchi D A, Elhabiri M, Goddard W A and Aprahamian I 2011 Isomerization mechanism in hydrazone-based rotary switches: lateral shift, rotation, or tautomerization? J. Am. Chem. Soc. 133 9812-23

[39] Durgun E and Grossman J C 2013 Photoswitchable molecular rings for solar thermal energy storage J. Phys. Chem. Lett. 4 $854-60$

[40] Soler J M, Artacho E, Gale J D, García A, Junquera J, Ordejoón P and Sánchez-Portal D 2002 The siesta method for $a b$ initio order-N materials simulation J. Phys. Condens. Matter 14 2745-79

[41] Perdew J P, Burke K and Ernzerhof M 1996 Generalized Gradient approximation made simple Phys. Rev. Lett. 77 3865-8

[42] Shi L, Fleming C J, Riechers S L, Yin N, Luo J, Lam K S and Liu G 2011 High-resolution imaging of dendrimers used in drug delivery via scanning probe microscopy J. Drug Deliv. 254095

[43] Yang G Y, Amro N A and Liu G 2003 Scaning probe lithography of self-assembled monolayers Proc. SPIE 5220 52-65

[44] Eggers P K, Zareie H M, Paddon-Row M N and Gooding J J 2009 Structure and properties of redox active self-assembled monolayers formed from norbornylogous bridges Langmuir 25 11090-96

[45] Coelho P J, Castro M C R and Raposo M M R 2013 Reversible trans-cis photoisomerization of new pyrrolidene heterocyclic imines J. Photochem. Photobiology A 259 $59-65$

[46] Lee N, Shuck P J, Nico P S and Gilbert B 2015 Surface enhanced Raman spectroscopy of organic molecules on magnetite $\left(\mathrm{Fe}_{3} \mathrm{O}_{4}\right)$ nanoparticles J. Phys. Chem. Lett. 6 970-74

[47] Socrates G 2001 Infrared and Raman Characteristic Group Frequencies (Chichester: Wiley)

[48] Sun M, Li Z, Liu Y and Xu H 2009 Direct visual evidence for chemical mechanisms of SERRS via charge transfer in $\mathrm{Au}_{20}$-pyrazine- $\mathrm{Au}_{20}$ junction J. Raman Spectrosc. 40 $1942-8$

[49] Gartia M R et al 2010 Rigorous surface enhanced Raman spectral characterization of large-area high-uniformity silvercoated tapered silica nanopillar arrays Nanotechnology $\mathbf{2 1}$ 395701-9 